\title{
Review
}

\section{The emerging role of RNAs in DNA damage repair}

\author{
Ben R Hawley ${ }^{1,2}$, Wei-Ting Lu ${ }^{1,2}$, Ania Wilczynska ${ }^{1}$ and Martin Bushell ${ }^{*, 1}$
}

Many surveillance and repair mechanisms exist to maintain the integrity of our genome. All of the pathways described to date are controlled exclusively by proteins, which through their enzymatic activities identify breaks, propagate the damage signal, recruit further protein factors and ultimately resolve the break with little to no loss of genetic information. RNA is known to have an integral role in many cellular pathways, but, until very recently, was not considered to take part in the DNA repair process. Several reports demonstrated a conserved critical role for RNA-processing enzymes and RNA molecules in DNA repair, but the biogenesis of these damage-related RNAs and their mechanisms of action remain unknown. We will explore how these new findings challenge the idea of proteins being the sole participants in the response to DNA damage and reveal a new and exciting aspect of both DNA repair and RNA biology.

Cell Death and Differentiation (2017) 24, 580-587; doi:10.1038/cdd.2017.16; published online 24 February 2017

Facts

- The miRNA biogenesis machinery has a role in DNA damage repair outside of canonical miRNA-mediated translational repression.

- RNA molecules have been observed in the proximity of DNA breaks and have been implicated in the DNA repair response.

- These phenomena have been observed in many species, indicating an evolutionarily conserved mechanism.

\section{Open Questions}

- What is the precise role of the RNA-processing enzymes in DNA repair?

- Do small RNAs have a direct mechanistic role in DNA repair, or do they serve as a by-product of a different RNA species?

- Is transcription induced locally at sites of DNA damage? Are proximal dormant promoter elements involved, or is an open-ended break sufficient for polymerase recruitment?

- Can these results be replicated outside of integrated exogenous reporter systems?

\section{An Unlikely Match: RNA Biogenesis Machinery Meets DNA Repair}

Our DNA is constantly exposed to various environmental and chemical agents, including ionising radiation (IR) from cosmic radiation, ultraviolet (UV) light from the sun or even nucleophilic attack induced by chemical compounds in food. ${ }^{1}$ In fact,
DNA damage is intrinsic to the process of life: it is inevitable during replication and essential during meiotic recombination. Also, controlled DNA breaks by topoisomerase occur to facilitate the resolution of supercoiled chromatin structures. Complex mechanisms have evolved to counteract the variety and quantity of DNA damage encountered daily.

Generally, DNA damage response (DDR) involves a complex signalling cascade initiated by one of three PI3Klike kinases: ATM, ATR or DNA-PK. They serve to facilitate chromatin modification and remodelling, allowing access to and acting as scaffolds for proteins involved in repair, as well as propagating the damage signal. ${ }^{1}$ Many of these recruited factors are involved in a binary decision-making process (see Figure 1). The repair of double-strand breaks (DSBs) is resolved by two distinct mechanisms: error-free homologous recombination (HR) or error-prone non-homologous endjoining (NHEJ). ${ }^{1,2}$ The choice of which mechanism is used can depend on chromosomal context and is cell cycle stage dependent; ${ }^{3} \mathrm{HR}$ is favoured when sister chromatids are available in G2 phase, whereas NHEJ is favoured over HR in the G1 stage of the cell cycle, and in resting or terminally differentiated cells. ${ }^{2,4}$ Commitment to the HR pathway is facilitated by the eviction of key repair proteins, such as 53BP1, from the damage site. ${ }^{2}$ This is followed by the recruitment of pro-HR proteins, such as BRCA1, FancD2 and CtIP, leading to the resection of DNA around break sites and the search of homologous chromatids for templatemediated repair. ${ }^{5}$ Conversely, stabilisation of 53BP1 at break sites by PTIP and Rif1 blocks resection, causing NHEJ to occur. $^{6,7}$

Traditionally, it has been thought that DNA repair involves only enzymatic reactions carried out by proteins that facilitate repair and propagate signalling events. Interestingly, a number

${ }^{1}$ MRC Toxicology Unit, Leicester, UK

${ }^{*}$ Corresponding author: M Bushell, MRC Toxicology Unit, Hodgkin Building, Leicester LE1 9HN, UK. Tel: +44 116252 5568; Fax: +44 116 252 5616;

E-mail: mb446@le.ac.uk

${ }^{2}$ These authors contributed equally to this work.

Received 15.11.16; revised 16.1.17; accepted 23.1.17; Edited by M Blagosklonny; published online 24.2.2017 


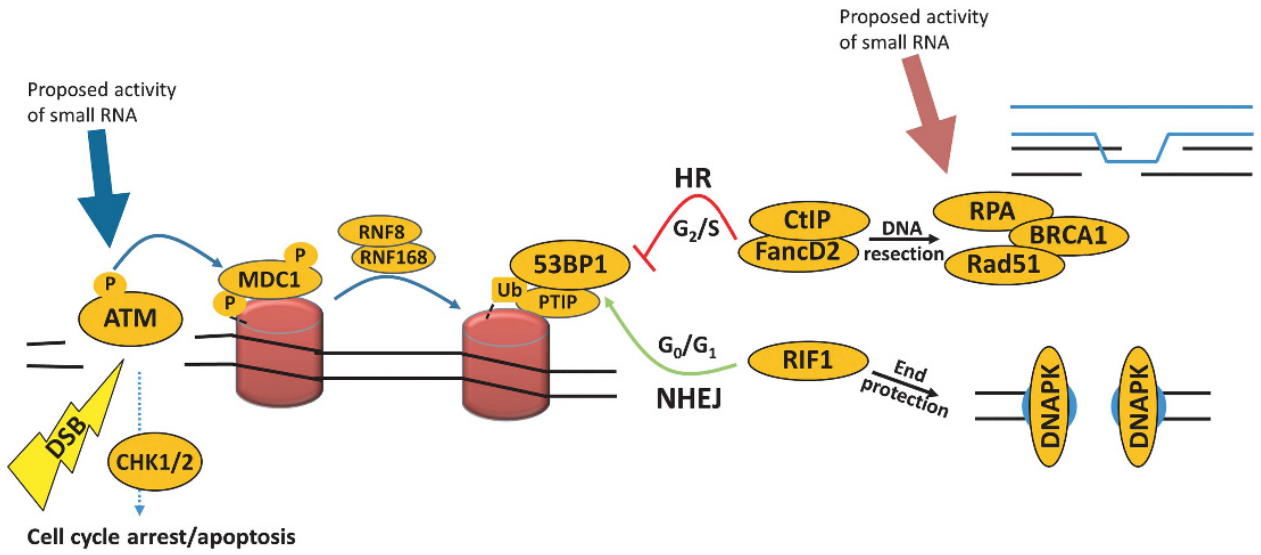

Figure 1 A schematic of the DNA repair pathway. The formation of a DSB induces the phosphorylation of ATM, which contributes to the activation of the DNA repair pathway and cell cycle arrest. A series of molecular signalling events lead to the deployment of ubiquitylation (Ub) marks on the histones (red cylinders) in the proximity of DNA breaks, facilitated by RNF8 and RNF168. The recruitment of 53BP1 marks the key crossroad of DSB repair (DSBR) pathway, which branches out into error-free HR or relatively errorprone NHEJ. Small RNAs have been proposed to function at two distinct steps in DSBR. Francia et al. ${ }^{11}$ suggested that it affects early signal propagation through ATM phosphorylation (blue arrow), while Gao et al. ${ }^{8}$ proposed that it only affects the HR sub-pathway via modulation of Rad51 binding (red arrow)

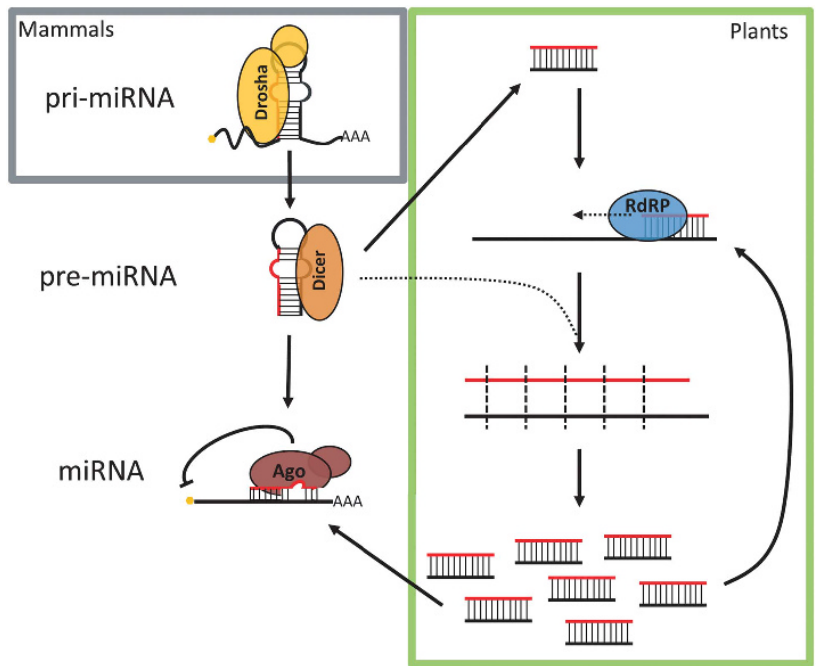

Figure 2 Outline of the microRNA biogenesis pathway in humans, and how plants utilise RdRPs to amplify these. The miRNA gene is transcribed by RNA polymerase II and typically capped and polyadenylated. This primary miRNA (primiRNA) contains the hairpin structure that is recognised and cleaved by Drosha, as part of the Microprocessor complex. The stem loop is then further trimmed by Dicer forming the pre-miRNA. In the canonical miRNA pathway, a single strand of the small RNA duplex is loaded into an Argonaute protein (Ago), which leads to repression of target transcripts. In plants, a dsRNA precursor is cleaved by Dicer into small dsRNA (green box). There exist multiple amplification pathways; broadly, an RdRP can synthesise a complementary strand by elongating a small RNA bound to its target RNA. Plant Dicer proteins can then cleave this newly generated dsRNA. to produce many secondary siRNAs that can repress target transcripts via Ago, or begin another cycle of small RNA amplification

of reports have now implicated RNA in DDR. ${ }^{8-10}$ These have largely concentrated on the involvement of the small RNA biogenesis enzymes (outlined in Figure 2) and have identified a novel species of small RNA, which appears to be derived from the vicinity of the DSB. The involvement of an RNA species in DDR is well-conserved evolutionarily with observations in fungi, yeast, plant, Drosophila and human cells. ${ }^{9,11-15}$
The first description came from the filamentous fungus $N$. crassa, where interplay between non-canonical small RNAs and the DDR was reported. Chemically induced replication stresses in $N$. crassa resulted in the production of small RNAs originating mostly from highly transcribed and repetitive ribosomal loci. This event was dependent on the presence of the fungal orthologue of Argonaute protein and an RNAdependent RNA polymerase. ${ }^{14}$ Although required for proficient DNA repair, these small RNAs appeared to be produced from the degradation of longer RNA species. ${ }^{14}$ The authors proposed that aberrant transcripts ('aRNA') transcribed as a result of DNA damage are amplified by RNA-dependent RNA polymerases (RdRPs) and processed into small RNA (termed quelling-induced RNA, qiRNA). These qiRNAs then act to degrade aRNA, in a manner similar to the siRNA amplification cycle. ${ }^{12,16,17}$ These aRNAs are transcribed from repetitive loci, such as the ribosomal DNA locus, and are refractory to RNA polymerase inhibitors. ${ }^{14}$ Interestingly, the production of aRNAs is dependent on the presence of replicating protein A, a known component of the HR repair pathway. ${ }^{18}$ How such a mechanism could aid in repair of a break itself is unclear. Nevertheless, one can imagine the suppression of these aberrant transcripts by qiRNA serves to complement nonsense-mediated decay to limit any possible translation of abnormal transcripts.

Similarly, production of small RNAs was observed postDNA damage in plants. The plant orthologs of Dicer protein, DCLs, are required for efficient DSB repair when $A$. thalina is challenged with $\mathrm{IR}^{9}{ }^{9}$ Utilising next-generation sequencing (NGS), it was shown that DNA damage-induced small RNAs (diRNAs) arose in the proximity of the DSB sites. Interestingly, although these diRNAs are required for proficient repair, they are not involved in the initial recognition of DSBs indicated by the continued phosphorylation of Histone H2A.X. ${ }^{9}$

Recently, the importance of diRNAs in the DDR pathway was highlighted in metazoa. Several publications have documented the requirement for small RNAs, or certain components of the small RNA biogenesis machinery, in proficient DNA repair signalling. ${ }^{8,10,11,13,19}$ It is largely agreed 
a

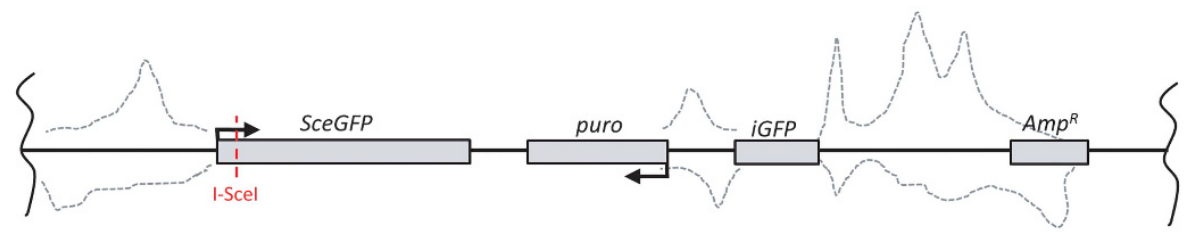

b

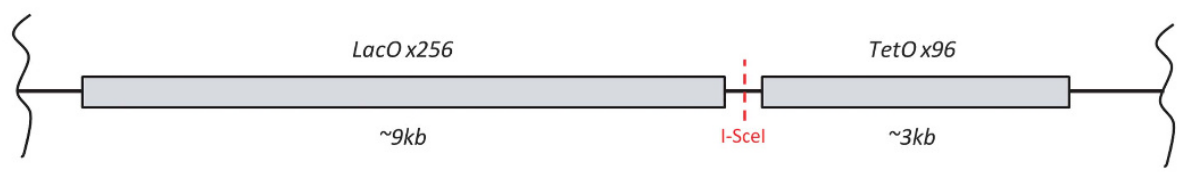

c
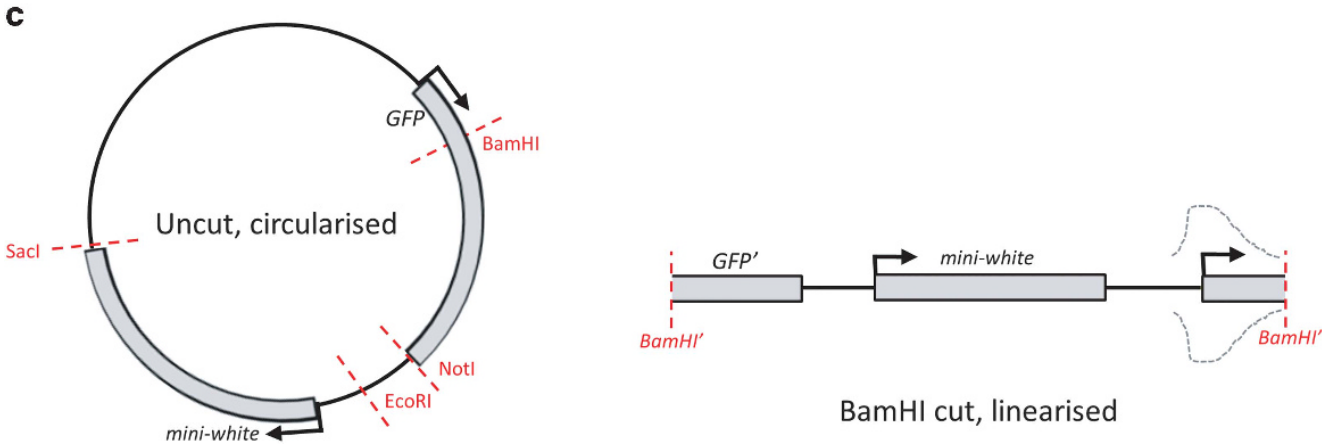

BamHI cut, linearised

Figure 3 Schematics of reporter systems used for small RNA sequencing experiments. (a) Wei et al. ${ }^{9}$ DR-GFP genomically integrated reporter. Transfection of I-Scel results in cleavage near the transcriptional start site (TSS) of GFP. Small RNA was sequenced and mapped back to the reporter sequence. (b) Francia et al..$^{11}$ genomically integrated Lac-/Tet-operator-flanked I-Scel site. This reporter lacks transcriptional activity but is highly repetitive. Small RNA was detected after I-Scel transfection but at low levels (47 total reads after transfection versus 20 reads without). No information on where these RNAs mapped to was provided. (c) Michalik et al. ${ }^{13}$ Drosophila expression plasmids, either circularised or linearised. Here only the BamHI linearised vector is shown as it produced the highest number of small RNA reads. Grey dashed lines represent the approximate distribution of small RNA mapping back to the locus, where positional data was supplied in the manuscript. Right-angled arrows represent TSS, whereas vertical wavy line denotes integration within the genome

that the key RNAse III family enzymes that process small RNA precursors, Drosha and Dicer, have a role in the DNA repair response. ${ }^{9-11}$ Indeed, the loss of diRNAs or the small RNA biogenesis machinery, appears to affect the DNA repair process and have an impact on repair pathway choice. .,10,16 $^{2}$ Which stage within the DDR pathway is affected by the loss of diRNAs and related proteins is currently contested (see Figure 1). Nevertheless, similarly to plants, it is thought that the initial phosphorylation of histone variant H2A.X is not affected by the loss of diRNAs. ${ }^{8,11}$ It is also noteworthy that RNA polymerase II activity has been implicated in this process. ${ }^{11}$

The generation of small RNA is a multistep biological process (Figure 2). Various accessory proteins such as DGCR8 are required alongside Dicer and Drosha, ${ }^{20}$ but their involvement in DNA repair has not been investigated in depth. Moreover, the participation of key downstream effectors in the RNAi pathway, namely the Argonautes, is also contested. ${ }^{8,10,11,21}$ Currently, the mechanism by which the diRNAs directly influence repair outcome is under extensive investigation. Nevertheless, it has been reported that in Drosophila cells, these small RNAs can serve as endosiRNAs to suppress existing transcripts arisen from the portion of DNA harbouring the DSB, as was proposed in N. crassa. ${ }^{13}$ Thus far, the evidence supporting the existence of DNA diRNAs has come from two main sources: deep sequencing and the isolation of the small RNA fraction for use in rescue experiments, which will be discussed in detail in this review.

\section{Search for the One: Using NGS to Identify diRNAs}

How can the generation of small RNA in a DNA damagespecific context be detected? Commonly used external DNA damage agents, such as IR, lead to the generation of multiple breaks at random genomic sites. This makes the task of discovery of novel RNA species with the use of an NGS approach virtually impossible, as breaks need to occur in known defined sequences for this experimental strategy to work. To date, three studies have used restriction enzymebased systems in animal cells combined with NGS to detect diRNAs, which map to the vicinity of the cut site. ${ }^{9,11,13}$ These reports relied on the ectopic expression of rare restriction enzymes targeted to specific pre-integrated loci in the genome. Two different systems were adopted in human cell lines: the DR-GFP HR reporter or a Lac-/Tet-operator repeatflanking reporter, ${ }^{22,23}$ both of which contain a single recognition site for the uniquely cutting meganuclease I-Scel (Figures $3 a$ and b, see also Figure 4 and the more in-depth discussion of the DR-GFP reporter assay below). Following the transfection and expression of I-Scel for 12 to $24 \mathrm{~h}$, small RNAs were sequenced by NGS. These two studies reported the existence of small RNAs around the break sites., ${ }^{9,11}$ 

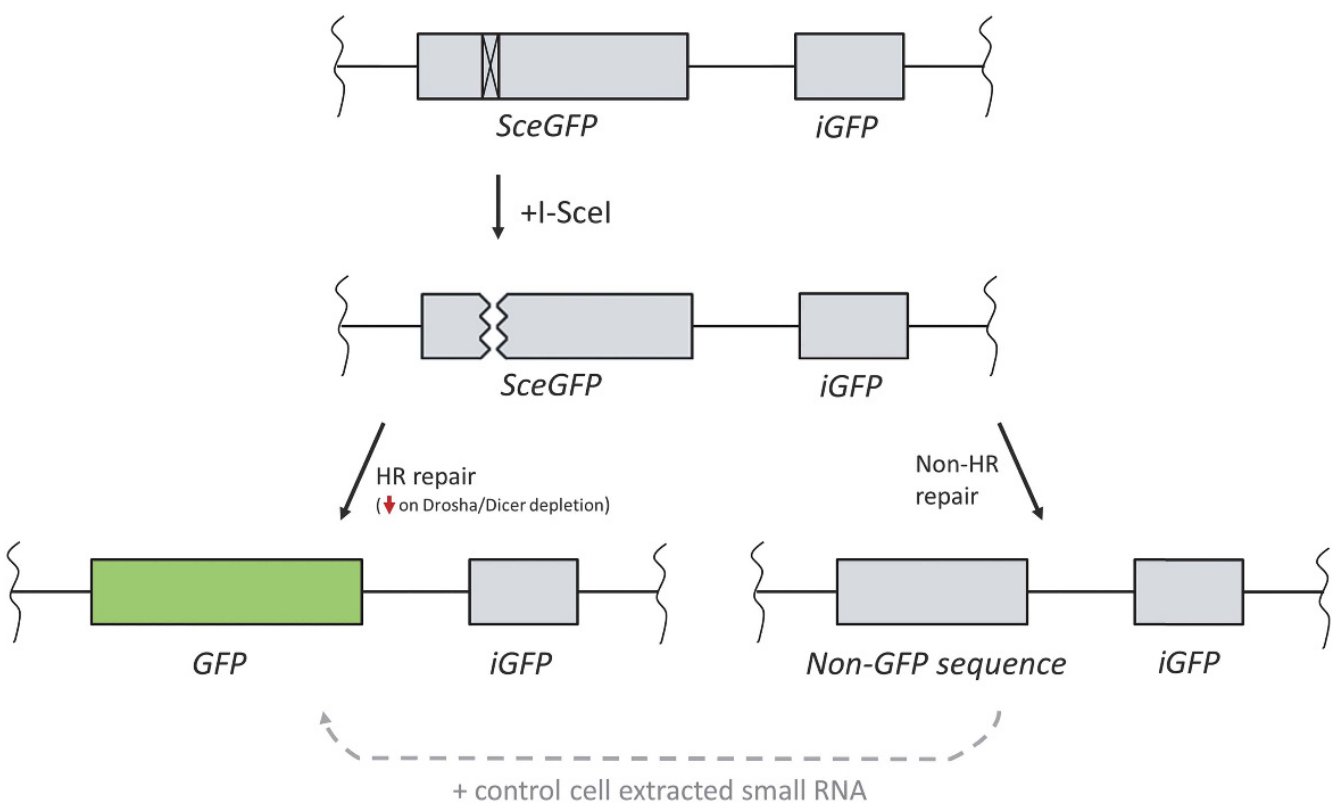

Figure 4 Detailed schematic of the DR-GFP HR repair reporter as in Figure 3a, used for RNA rescue experiments. A copy of the reporter is integrated into the genome to provide appropriate chromatin context. Insertion of an I-Scel restriction site within the GFP ORF results in a nonsense product that will produce no green fluorescence. After the induction of I-Scel cleavage, the cell can repair the resulting DSB via HR using the downstream internal GFP sequence (iGFP), producing a full-length GFP product. If HR is impaired, the break will instead be repaired via an alternate pathway, such as NHEJ, resulting in a sequence lacking full GFP coding region. The extent of deletion is dependent upon the non-HR mechanism chosen by the cell, but any loss of sequence within the I-Scel restriction site will prevent any further cutting. In the experiments by Wei et al. ${ }^{9}$ and Wang and Goldstein, ${ }^{10}$ the loss of Drosha and Dicer resulted in a lack of GFP indicating a deficiency in HR repair; however, when small RNAs extracted from control cells were incubated with these deficient cells for $1 \mathrm{~h}$, GFP was found to be expressed (denoted by dashed arrow)

However, the exact roles of these small RNAs are yet to be determined.

An alternative approach has involved the transfection of either circular (uncut) or linearised (cut) plasmids into Drosophila S2 cells (Figure 3c). ${ }^{23}$ Similarly, it was found that small RNA can be generated from the vicinity of DNA break sites. Strikingly, these small RNAs can be generated in response to either blunt or staggered DNA ends and they proceed to serve as endo-siRNAs to repress corresponding transcripts in trans. ${ }^{13}$ It is noteworthy that this response can only be provoked by a DNA DSB, but not a nicked DNA. ${ }^{13} \mathrm{~A}$ recent follow-up article by the same group utilised a similar GFP-based reporter to that previously described ${ }^{13}$ and again reported small RNAs mapping to the damaged locus following damage induction. ${ }^{2,13,24}$ In summary, these reports by Forstemann and co-workers suggest that diRNA function in Drosophila appears to be more similar to plant-based qiRNAs, which remove aberrant transcripts or aid in other RNA metabolic processes. ${ }^{13,14,24}$ In contrast, in mammalian cells, they are reported to have a direct contribution to the DNA repair processes. ${ }^{10,11}$

Although there are many differences between the several studies published thus far, it is important to note that all the groups have found an enrichment of small RNAs mapping to exogenous loci following DNA damage. It is clear from these studies that the miRNA biogenesis enzymes and RNA species are critically involved in the DNA repair process with multiple groups presenting similar observations in a diverse collection of experimental settings. Here, we discuss these results and examine the merit of the different approaches taken, integrating them to develop new hypotheses for how RNA could participate in DNA repair.

\section{Needles in a Haystack: Methodologies and Difficulties of diRNA Discovery by NGS}

The advent of NGS has revolutionised the RNA field, allowing robust quantitation of RNA changes between samples and discovery of novel transcripts and splice variants in a highthroughput manner. As such, NGS was the sensible choice for discovering novel RNA species at DSBs.

These small RNAs were first identified by NGS in plants and humans. ${ }^{9,11}$ Reads mapping to regions proximal to the integration locus of the HR repair reporter were observed, with the earliest peaks of RNA reads visualised $12 \mathrm{~h}$ following the appearance of DSB induced by the transfection of I-Scel (Figure 3a). ${ }^{25}$ Interestingly, the bulk of these mapped RNAs do not appear to have arisen directly from the DNA break site. Instead, they were mapped to the sequence upstream of the start site of the cut GFP gene, located upstream of the puromycin resistance gene, or downstream of the homologous GFP sequence. ${ }^{9}$ It thus appears that the small RNAs are mapped to highly transcribed regions proximal to the break site, making it unclear as to whether they are true de novo transcripts, or degradation products of pre-existing mRNAs. Small RNAs were also shown to be generated in plants postDNA damage, with read numbers considerably higher than those in human cells. ${ }^{9}$ One aspect of plant biology that may contribute to this difference is the existence of RdRPs (Figure 2, green box). It is possible that after DNA damage, 


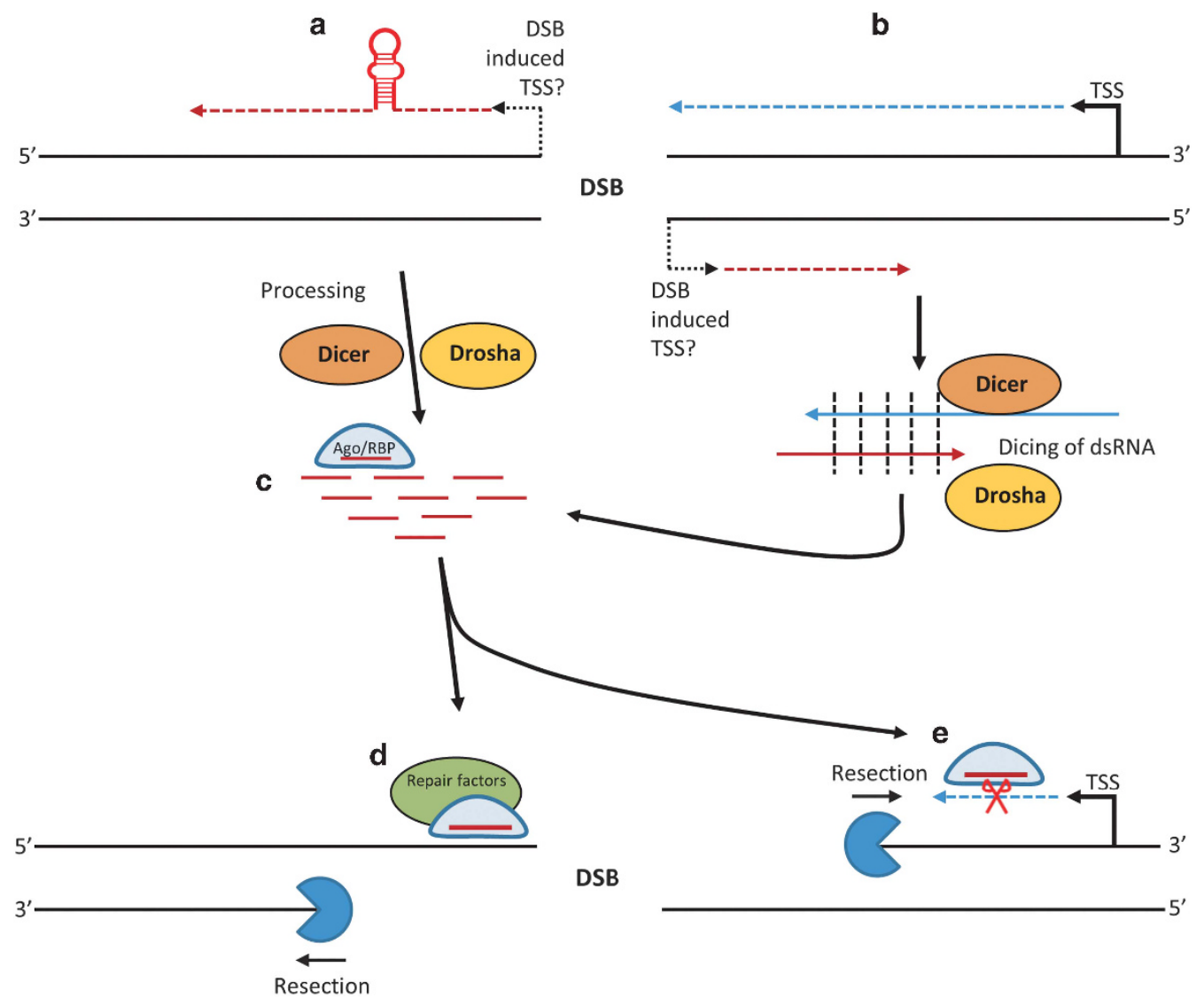

Figure 5 Postulated biogenesis of diRNAs and their function in DDR. Directionality of transcription, transcripts, and resection is 5' to 3', denoted by arrows. (a) A new transcript is formed from the break site. Any secondary structure formed by this transcript that can be recognised by Drosha and Dicer is processed into small RNAs, mirroring miRNA generation. (b) Where a cut occurs within an actively transcribing gene, a new end-dependent transcription event could take place resulting in formation of an antisense RNA. These RNA species can anneal to form dsRNA, and are then diced into small RNA substrates by Dicer. (c) The resulting small RNAs could then be incorporated into an Argonaute or another RBP, to carry out diRNA functions, which are hypothesised to be: (d) recruitment of repair and chromatin remodelling factors to the site of damage, (e) degradation of potentially aberrant transcripts or other as-of-yet unexplored functions

RdRPs may be activated to amplify nascent transcripts into dsRNAs that are then further processed into diRNAs by Dicer, in a similar manner to that reported in $N$. crassa. Interestingly, the authors showed that RNA pol IV, which is responsible for transcription from repetitive and transposable elements, was critical for the production of diRNAs in plants and loss of this enzyme significantly reduced repair efficiency. ${ }^{9}$ In plants, RDR2 is the RdRP responsible for amplifying pol IV transcripts to produce the hc-siRNA class of small RNAs. ${ }^{26,27}$ When RDR2 was ablated, the number of diRNAs was hugely reduced, however, repair efficiency was unaffected. ${ }^{9}$ This discrepancy between overall small RNA levels and repair efficiency suggests that the nascent RNAs produced following damage are important for repair resolution, but perhaps the amplification of secondary RNA products by RDR2 is not. This could represent a distinction in the role of small RNAs between plants and metazoa, where in animals an amplification loop is not required for a secondary role for functional RNA molecules. Alternatively, a similar mechanism may be utilising a yet undiscovered RdRP activity in animals. For example, in humans TERT-RMRP and RNA polymerase II have been demonstrated to have slight RdRP activity. ${ }^{28-30}$
As these reporter systems are under the control of viral promoters with high basal activity, it is entirely plausible that the pre-existing, highly abundant long RNAs transcribed from reporter loci are degraded as part of the DDR. ${ }^{31}$ The approach taken by Francia et al. ${ }^{11}$ partially addresses this possibility: the Tet-/Lac-flanked I-Scel sequence used is devoid of any transcriptional elements and thus any new RNAs should be generated in a DNA damage-dependent manner (see Figure $3 b$ ). Following deep sequencing, they reported a total of 47 reads arising from the $12 \mathrm{~kb}$ integrated locus when cut with the endonuclease, compared with 20 in uncut controls. As the parental cell lines produced no small RNAs that mapped to this sequence, these small RNAs are indeed sequencespecific and dependent on reporter integration. ${ }^{11}$ However, with the low read counts, and modest enrichment above background level, it is hard to convincingly conclude that new RNA species are specifically transcribed post-damage. It should also be noted that the presence of the 20 small RNA reads in the absence of damage suggests these RNAs may not be entirely damage dependent.

When similar deep sequencing investigations were performed in cells depleted of Drosha and Dicer, the analyses revealed that only loss of Dicer reduced small RNA counts 
significantly. ${ }^{11}$ The lack of a role for Drosha in production of the small RNAs but the requirement for Dicer suggests that diRNAs may be produced from the cleavage of a longer dsRNA precursor rather than from any pri-miRNA-like secondary structures within an ssRNA precursor (see also Figure 5 part $a$ and $b$ ). It is important to remember that Drosha has been observed to impact DNA repair efficiency. ${ }^{11}$ This may echo the previously discussed observation in plants that generation of the small RNAs (by pol IV and RDR2) was unconnected to repair outcome. It should also be noted that Dicer and Drosha are known to have a role in non-canonical termination of new RNA transcripts. ${ }^{19,32,33}$ Whether this activity of Drosha is utilised after DNA damage warrants further investigation.

Using a comparatively straightforward system, Michalik et al. ${ }^{13}$ transfected Drosophila S2 cells with several exogenous sequences: a GFP expression vector and an unrelated yeast plasmid, which were either linearised ('cut') or circularised ('uncut') (see Figure 3c). A small linear PCR amplicon comprising firefly luciferase coding sequence was also used as an additional control. Following deep sequencing, small RNAs were mapped to the vector sequences with significantly more reads arising from the linearised Drosophila vector than the circularised one. Depending on the restriction enzyme used to generate the linearised plasmids, different patterns of small RNA were produced. This suggests the context of the DSB may affect the pattern of newly transcribed RNA. The small RNAs appeared to map predominantly upstream of the cut site, with the majority of small RNAs arising from regions adjacent to the GFP promoter. Similar to the studies conducted by Francia et al. ${ }^{11}$ and Wei et al., ${ }^{9}$ these data do not distinguish between nonspecific degradation of RNA produced from the reporter as a result of recognition of DSBlike structures, or a deliberate processing event that generates small RNAs that may have a direct mechanistic role in DNA repair. ${ }^{13}$ Intriguingly, the lack of reads mapping to the PCR product, but a surprisingly high number of reads for the control yeast plasmid, suggests that some potential promoter activity may be required. One may argue that a possible pitfall of this plasmid-based approach is that the cell is exposed to DNA lacking any chromatin structure. Thus, it is hard to relate these observations to DNA damage within a genomic context. Also, it is possible that these sequences are generated in response to introduction of foreign genetic material by an anti-viral or retrotransposon defence mechanism independent of the DDR. $^{34-36}$ However, the lack of a response from the transfected control PCR product suggests this is not the case.

Considering all these limitations, we propose that the ideal experimental setting to investigate the existence of diRNAs requires a system that produces DSBs at a range of different sites within the genome. This way, the potential involvement of chromatin structure or transcriptional status can be investigated. Thus far, two endogenous restriction enzyme-based systems have been extensively utilised in the DDR field: AsiSI $^{3,37,38}$ and I-Ppol. ${ }^{10,39}$ Alternatively, the CRISPR-Cas9 system also allows the induction of DSBs at specific sites of the genome: ${ }^{40,41}$ using specially designed guide RNAs, the dynamics of diRNA production could be investigated even further by comparing DSBs generated proximal with promoters and transcriptional start sites to those generated further away within the same gene. Such approaches could help elucidate whether newly produced RNAs arise from transcription events at the DNA break site, or from a promoter or cryptic promoter in the vicinity of the DSBs.

\section{To Mend a Broken Heart: Pre-isolated Small RNA Fraction Acts in DNA Repair}

The major alternative strategy used to investigate the existence of small RNAs produced following DNA damage involved the isolation of the small RNA from cells and delivery of that RNA into cells that lack the ability to produce them. Cell lines carrying a DR-GFP integrated reporter (Figure 4) were incubated with a pre-extracted small RNA fraction from damaged or undamaged cells. ${ }^{9,11}$ This reporter consists of a GFP open reading frame containing an inserted I-Scel recognition site, which when transcribed results in an aberrant transcript. I-Scel induction leads to cleavage at the nonfunctional GFP, and allows repair via HR using the downstream intact sequence as template. This results in the creation of a copy of GFP that can express a full-length protein. The outcome of HR repair can then be measured by analysing GFP-positive cells by flow cytometry.

Depletion of Drosha or Dicer in cells carrying this transgene following 2 days of damage resulted in a reduction in GFPpositive cells indicating that HR was impaired. ${ }^{9,10}$ Interestingly, two groups reported that incubation with small RNAs isolated from previously damaged cells for just $1 \mathrm{~h}$ could restore HR efficiency. ${ }^{8,10}$ In contrast, small RNAs extracted from undamaged cells failed to accomplish such rescue. ${ }^{10}$ This suggests the involvement of an RNA species with sequence-specific characteristics in the DDR process. The processes of transcription through to translation of a gene can take from minutes to hours, while the process of maturation and folding of fluorescent proteins may take even longer. ${ }^{42,43}$ Therefore, it is unexpected that the incubation of small RNAs for merely an hour, days after the induction of DNA damage at GFP loci, can rescue expression of GFP within such a short timeframe, especially when HR repair only occurs during the S/G2-phase of the cell cycle. Moreover, given the nature of these experiments, if repair of cut sites is carried out by an errorprone non-HR mechanism, mutations will be introduced into the cut site preventing subsequent cleavage events (see Figure 4). Therefore, although it is possible for this to occur, it is unclear whether incubation with small RNAs $1 \mathrm{~h}$ before FACS analysis can result in restoration of functional $\mathrm{HR}$ repair at this specific break site.

Nevertheless, it is important to remember that this method was not the only approach used by the authors. Small RNAs were isolated from damaged cells and were able to restore 53BP1 DDR foci in cells pre-treated with RNase A. ${ }^{11}$

\section{Concluding Remarks and Future Perspectives}

Recent advances in deep sequencing have made it possible to conduct refined experiments leading to the suggestion of involvement of human small RNA processing machinery in DDR. This is especially interesting given certain reports demonstrating that RNA molecules may be used as templates for DNA repair in yeast. ${ }^{12}$ Although it is largely agreed that 
Dicer and Drosha have some role in DNA repair, its mechanism is still elusive (see Figure 5). ${ }^{8-11}$ Also, it is not clear whether this mechanism involves the typical co-factors of Drosha and Dicer, such as DGCR8, DDX5, DDX17 and TRBP. Given that Dicer and Drosha are involved in the non-canonical termination of transcription and modulation of RNA polymerase II activity, it is possible that certain interaction partners may not be required for the DNA repair-related activity. ${ }^{19,32,33}$

One primary direction for further investigation is the identity and biogenesis mechanism of the RNA species involved in DNA repair: whether these species are bona fide new small RNA transcripts derived from the vicinity of the break site, or degradation products of pre-existing transcripts. Whether they can serve as RNA templates (or remnants of RNA templates) that actively participate in the repair process is unknown. However, it should be noted that while several classes of noncoding RNA are produced in association with DNA damage, the impact of these RNAs on the repair process in different model systems is varied (see Table 1). For example, plantbased aRNAs, qiRNAs and hc-siRNA require RdRP activity, and they have been shown to induce the degradation of transcripts. ${ }^{14,18,26}$ Similarly, diRNAs are reported to serve as endo-siRNAs in Droshophila systems. ${ }^{13,24}$ However, the small RNA produced in mammalian cells, termed diRNAs, are reported to be directly involved in the repair process, but the mechanism of action is still under debate. ${ }^{8-11}$

Recent studies have provided the first direct evidence for an RNA-templated repair mechanism in both yeast and human cells, the latter of which curiously utilises the NHEJ machinery. ${ }^{12,44}$ Again in yeast, an even more recent paper also demonstrates the formation of RNA:DNA hybrids at sites of DNA damage, showing a strong link between transcription and DNA repair. ${ }^{45}$ Alternatively, they may also be involved in the process of modulating chromatin states, in a manner similar to piwi-interacting RNA in germ cells. ${ }^{46}$ Provided that these small RNAs function in a sequence-specific manner analogous to RNAi, one should expect that an Argonaute-like protein would be required to facilitate scanning and base pairing with its genomic target (Figure 5). ${ }^{47}$ Currently, the jury is still out regarding the exact role of Ago2 protein in DDR..$^{8,10,21}$

With recent reports documenting crosstalk between the DNA repair processes and RNA transcription, processing and splicing machinery, one can only envisage an even more intertwined interaction between RNA and the DNA repair process. $^{3,25,48}$

\section{Conflict of Interest}

The authors declare no conflict of interest.

Acknowledgements. The authors are funded by the Medical Research Council (MRC), UK. AW is supported by the BBSRC and W-TL was partly supported by MRC Centenary Award. We thank Gaelle Legube (CNRS, University of Toulouse III) for critical reading of the manuscript.

1. Ciccia A, Elledge SJ. The DNA damage response: making it safe to play with knives. Mol Cell 2010; 40: 179-204.

2. Chapman JR, Taylor MR, Boulton SJ. Playing the end game: DNA double-strand break repair pathway choice. Mol Cell 2012; 47: 497-510. 
3. Aymard F, Bugler B, Schmidt CK, Guillou E, Caron P, Briois S et al. Transcriptionally active chromatin recruits homologous recombination at DNA double-strand breaks. Nat Struct Mol Biol 2014; 21: 366-U172.

4. Lu WT, Lemonidis K, Drayton RM, Nouspikel T. The Fanconi anemia pathway is downregulated upon macrophage differentiation through two distinct mechanisms. Cell Cycle 2011; 10: 3300-3310.

5. Unno J, Itaya A, Taoka M, Sato K, Tomida J, Sakai W et al. FANCD2 binds CtIP and regulates DNA-end resection during DNA interstrand crosslink repair. Cell Rep 2014; 7: 1039-1047.

6. Panier S, Boulton SJ. Double-strand break repair: 53BP1 comes into focus. Nat Rev Mol Cell Biol 2014; 15: 7-18

7. Zimmermann M, de Lange T. 53BP1: pro choice in DNA repair. Trends Cell Biol 2014; 24: 108-117.

8. Gao M, Wei W, Li MM, Wu YS, Ba Z, Jin KX et al. Ago2 facilitates Rad51 recruitment and DNA double-strand break repair by homologous recombination. Cell Res 2014; 24: 532-541.

9. Wei W, Ba ZQ, Gao M, Wu Y, Ma YT, Amiard S et al. A role for small RNAs in DNA doublestrand break repair. Cell 2012; 149: 101-112.

10. Wang Q, Goldstein M. Small RNAs recruit chromatin-modifying enzymes MMSET and Tip60 to reconfigure damaged DNA upon double-strand break and facilitate repair. Cancer Res 2016; 76: 1904-1915.

11. Francia S, Michelini F, Saxena A, Tang D, de Hoon M, Anelli V et al. Site-specific DICER and DROSHA RNA products control the DNA-damage response. Nature 2012; 488: 231-235.

12. Keskin $H$, Shen $Y$, Huang F, Patel M, Yang T, Ashley $K$ et al. Transcript-RNA-templated DNA recombination and repair. Nature 2014; 515: 436-439.

13. Michalik KM, Bottcher R, Forstemann K. A small RNA response at DNA ends in Drosophila. Nucleic Acids Res 2012; 40: 9596-9603.

14. Lee HC, Chang SS, Choudhary S, Aalto AP, Maiti M, Bamford DH et al. qiRNA is a new type of small interfering RNA induced by DNA damage. Nature 2009; 459: 274-277.

15. Hall IM, Noma K, Grewal SI. RNA interference machinery regulates chromosome dynamics during mitosis and meiosis in fission yeast. Proc Natl Acad Sci USA 2003; 100: 193-198.

16. Storici F, Bebenek K, Kunkel TA, Gordenin DA, Resnick MA. RNA-templated DNA repair. Nature 2007; 447: 338-341.

17. Ghildiyal M, Zamore PD. Small silencing RNAs: an expanding universe. Nat Rev Genet 2009; 10: 94-108.

18. Lee HC, Aalto AP, Yang Q, Chang SS, Huang G, Fisher D et al. The DNA/RNA-dependent RNA polymerase QDE-1 generates aberrant RNA and dsRNA for RNAi in a process requiring replication protein $A$ and a DNA helicase. PLOS Biol 2010; 8: 10.

19. Castel SE, Ren J, Bhattacharjee S, Chang AY, Sanchez M, Valbuena A et al. Dicer promotes transcription termination at sites of replication stress to maintain genome stability. Cell 2014; 159: 572-583

20. Finnegan EF, Pasquinelli AE. MicroRNA biogenesis: regulating the regulators. Crit Rev Biochem Mol Biol 2013; 48: 51-68.

21. Gioia U, d'Adda di Fagagna F. Human nuclear ARGONAUTE 2 interacts in vivo only with small RNAs and not with DNA. Cell Cycle 2015; 14: 2001-2002.

22. Soutoglou E, Dorn JF, Sengupta K, Jasin M, Nussenzweig A, Ried T et al. Positional stability of single double-strand breaks in mammalian cells. Nat Cell Biol 2007; 9: 675-U121.

23. Pierce AJ, Johnson RD, Thompson LH, Jasin M. XRCC3 promotes homology-directed repair of DNA damage in mammalian cells. Gene Dev 1999; 13: 2633-2638.

24. Schmidts I, Bottcher R, Mirkovic-Hosle M, Forstemann K. Homology directed repair is unaffected by the absence of siRNAs in Drosophila melanogaster. Nucleic Acids Res 2016; 44: 8261-8271.

25. Beli P, Lukashchuk N, Wagner SA, Weinert BT, Olsen JV, Baskcomb L et al. Proteomic investigations reveal a role for RNA processing factor THRAP3 in the DNA damage response. Mol Cell 2012; 46: 212-225.

26. Haag JR, Ream TS, Marasco M, Nicora CD, Norbeck AD, Pasa-Tolic $L$ et al. In vitro transcription activities of Pol IV, Pol V, and RDR2 reveal coupling of Pol IV and RDR2 for dsRNA synthesis in plant RNA silencing. Mol Cell 2012; 48: 811-818.

27. Chapman EJ, Carrington JC. Specialization and evolution of endogenous small RNA pathways. Nat Rev Genet 2007; 8: 884-896.

28. Maida $\mathrm{Y}$, Yasukawa M, Furuuchi $\mathrm{M}$, Lassmann $\mathrm{T}$, Possemato $\mathrm{R}$, Okamoto $\mathrm{N}$ et al. An RNA-dependent RNA polymerase formed by TERT and the RMRP RNA. Nature 2009; 461: 230-U104.
29. Lehmann E, Brueckner F, Cramer P. Molecular basis of RNA-dependent RNA polymerase II activity. Nature 2007; 450: 445-449.

30. Wagner SD, Yakovchuk P, Gilman B, Ponicsan SL, Drullinger LF, Kugel JF et al. RNA polymerase II acts as an RNA-dependent RNA polymerase to extend and destabilize a noncoding RNA. EMBO J 2013; 32: 781-790.

31. Thomas MP, Liu X, Whangbo J, McCrossan G, Sanborn KB, Basar E et al. Apoptosis triggers specific, rapid, and global mRNA decay with $3^{\prime}$ uridylated intermediates degraded by DIS3L2. Cell Rep 2015; 11: 1079-1089.

32. Dhir A, Dhir S, Proudfoot NJ, Jopling CL. Microprocessor mediates transcriptional termination of long noncoding RNA transcripts hosting microRNAs. Nat Struct Mol Biol 2015; 22: 319.

33. Gromak N, Dienstbier M, Macias S, Plass M, Eyras E, Caceres JF et al. Drosha regulates gene expression independently of RNA cleavage function. Cell Rep 2013; $\mathbf{5}$ 1499-1510.

34. Soifer HS, Zaragoza A, Peyvan M, Behlke MA, Rossi JJ. A potential role for RNA interference in controlling the activity of the human LINE-1 retrotransposon. Nucleic Acids Res 2005; 33 846-856.

35. Yang N, Kazazian HH Jr. L1 retrotransposition is suppressed by endogenously encoded small interfering RNAs in human cultured cells. Nat Struct Mol Biol 2006; 13: 763-771.

36. Campo S, Gilbert KB, Carrington JC. Small RNA-based antiviral defense in the phytopathogenic fungus Colletotrichum higginsianum. PLoS Pathogens 2016; 12: e1005640.

37. lacovoni JS, Caron P, Lassadi I, Nicolas E, Massip L, Trouche D et al. High-resolution profiling of gamma $\mathrm{H} 2 \mathrm{AX}$ around DNA double strand breaks in the mammalian genome. EMBO J 2010; 29: 1446-1457.

38. Massip L, Caron P, lacovoni JS, Trouche D, Legube G. Deciphering the chromatin landscape induced around DNA double strand breaks. Cell Cycle 2010; 9: 2963-2972.

39. Berkovich E, Monnat RJ, Kastan MB. Roles of ATM and NBS1 in chromatin structure modulation and DNA double-strand break repair. Nat Cell Biol 2007; 9: 683-U137.

40. Jinek M, East A, Cheng A, Lin S, Ma E, Doudna J. RNA-programmed genome editing in human cells. eLife 2013; 2: e00471.

41. Mali P, Yang L, Esvelt KM, Aach J, Guell M, DiCarlo JE et al. RNA-guided human genome engineering via Cas9. Science 2013; 339: 823-826.

42. Jonkers I, Kwak H, Lis JT. Genome-wide dynamics of Pol II elongation and its interplay with promoter proximal pausing, chromatin, and exons. eLife 2014; 3: e02407.

43. lizuka R, Yamagishi-Shirasaki M, Funatsu $T$. Kinetic study of de novo chromophore maturation of fluorescent proteins. Anal Biochem 2011; 414: 173-178.

44. Chakraborty A, Tapryal N, Venkova T, Horikoshi N, Pandita RK, Sarker AH et al. Classical non-homologous end-joining pathway utilizes nascent RNA for error-free double-strand break repair of transcribed genes. Nat Commun 2016; 7: 13049.

45. Ohle C, Tesorero R, Schermann G, Dobrev N, Sinning I, Fischer T. Transient RNA-DNA hybrids are required for efficient double-strand break repair. Cell 2016; 167: 1001-1013 e1007.

46. Siomi MC, Sato K, Pezic D, Aravin AA. PIWl-interacting small RNAs: the vanguard of genome defence. Nat Rev Mol Cell Biol 2011; 12: 246-258.

47. Salomon WE, Jolly SM, Moore MJ, Zamore PD, Serebrov V. Single-molecule imaging reveals that argonaute reshapes the binding properties of its nucleic acid guides. Cell 2015; 162: 84-95.

48. Savage KI, Gorski JJ, Barros EM, Irwin GW, Manti L, Powell AJ et al. Identification of a BRCA1-mRNA splicing complex required for efficient DNA repair and maintenance of genomic stability. Mol Cell 2014; 54: 445-459.

(i) This work is licensed under a Creative Commons Attribution 4.0 International License. The images or other third party material in this article are included in the article's Creative Commons license, unless indicated otherwise in the credit line; if the material is not included under the Creative Commons license, users will need to obtain permission from the license holder to reproduce the material. To view a copy of this license, visit http:/l creativecommons.org/licenses/by/4.0/

(C) The Author(s) 2017 